\title{
Mesio-distal crown width in permanent dentition amongst adolescent population of Province II of Nepal
}

\author{
Alok Kumar Jaiswal ${ }^{1 *}$ (D), Umesh Parajuli ${ }^{2}$ (D), Manish Bajracharya ${ }^{3}$, Binita Singh ${ }^{1}$ \\ Department of Orthodontics and Dentofacial Orthopaedics, 'M B Kedia Dental College and teaching hospital, Birgunj, Nepal, \\ ${ }^{2}$ Gandaki Medical College, Pokhara ${ }^{3}$ National Academy of Medical Sciences, Bir Hospital, Kathmandu
}

\begin{abstract}
Background: The purpose of this study was to gather normative data on the mesio-distal crown dimensions amongst adolescent population of Province II, Nepal, to make an accurate diagnosis and treatment planning in orthodontics. It will also be useful in various clinical disciplines of dentistry including basic dental and anthropological research. Materials and Methods: Samples were selected Full form OPD of M B Kedia Dental College, Birgunj, Nepal. Total numbers of participants were 120, out of which 60 males and 60 females were selected after initial examination aged between 11 to 23 years. Subjects with all permanent teeth erupted (except second and third molar) without any history of previous orthodontic treatment and with no dental anomalies were included in this study. The alginate impressions were made by the well trained dental surgeon. Digital vernier calliper providing measurements to \pm 0.01 millimeter(mm) was used to measure the mesio-distal dimension of all teeth. Results: The mean, range and standard deviation were calculated for the size of the teeth. Independent $t$-test was used to compare between male and female population. The significance level was set at $p$ value $<=0.05$. The population of Province II, Nepal shows greater sexual dimorphism in mesio-distal crown dimension which was exhibited by the maxillary molars $(0.88 \mathrm{~mm})$ followed by mandibular molars $(0.38 \mathrm{~mm})$. Similarly in anterior tooth segment the maxillary canines $(0.29 \mathrm{~mm})$ followed by the mandibular canines $(0.27 \mathrm{~mm})$. Conclusion: The mean mesio-distal crown dimensions of the permanent dentition of males were larger than that of females for each type of tooth except maxillary central and lateral incisor.
\end{abstract}

Keywords: adolescent, mesio-distal width, sexual dimorphism

\section{*Corresponding Author}

Dr Alok Kumar Jaiswal

Associate Professor, Department of Orthodontics and Dentofacial Orthopaedics, ${ }^{1}$ M B Kedia Dental College and teaching hospital, Birgunj, Nepal,

Email: dralokjaiswal@gmail.com

Submitted: April 27, 2020

Accepted: June 18, 2020

To cite: Jaiswal AK, Parajuli U, Bajracharya M, Singh B. Mesio-distal crown width in permanent dentition amongst adolescent population of Province II of Nepal. JGMC Nepal. 2020;13(1):51-55. DOI: 10.3126/jgmcn. v13i1.28629

\section{INTRODUCTION}

Variation of tooth size is influenced by genetic and environmental factors. Many studies reported that variation of tooth size exists within the race and in subjects with different racial origin. ${ }^{1}$ According to Proffit and Fields ${ }^{2}$, tooth space analysis should be done accurately before any Orthodontic treatment. Tooth size discrepancy is defined as a disproportion among the sizes of individual teeth and amongst the jaws. ${ }^{2}$ In order to achieve optimal occlusion maxillary to mandibular tooth width ratios must be proportionate in size.

Many authors have evaluated factors associated with differences in the tooth width. The relationship between malocclusion type and tooth size proportions has also been reported. ${ }^{1,3-7}$ Since differences in tooth size have been reported with ethnic groups, ${ }^{3,8-11}$ most of the practitioners now disagree to apply the tooth size reported from the other ethnic groups. Bishara et al. ${ }^{11}$ conducted a study which includes the population of Egypt, Mexico, and the United States. They 
noticed that there was a difference in the mesio-distal dimensions of teeth among the three populations. There was sexual dimorphism where males had larger teeth than females in all three groups of population. There were no statistical significant differences between the right and left sides for all the three study groups.

Till date, few studies on the mesio-distal dimensions of teeth of the Nepalese population are found in the literature. No such studies have been reported in the population of province II. This study was carried out to gather data from province II on the mesio-distal widths of the permanent teeth and to see the gender differences in tooth width. This will help Orthodontist to diagnose the case properly and finish the case with precise occlusion.

\section{MATERIALS AND METHODS}

This was a hospital based cross-sectional study conducted at M B Kedia Dental College, Birgunj, Nepal, within the duration of August 2018 to November 2018. The study was conducted after getting ethical clearance from institutional review board, M B Kedia Dental College Birgunj, Nepal. Sample size calculation was based on $80 \%$ power and significance level of $5 \%{ }^{12}$ and considering 0.70 as maximum tolerable error rate and based on standard deviation of $2.5 . \mathrm{N}=[\mathrm{Z}+(1-\beta)]^{2} \mathrm{X} \mathrm{SD}{ }^{2} / \mathrm{L}^{2}$, where, $\mathrm{Z}=$ Confidence interval $(95 \%, \mathrm{CI}=1.96), \quad \beta=$ probability of type II error $=0.16$, Standard Deviation $=2.5, \mathrm{~L}=$ tolerable error $=0.70$ and $\mathrm{N}=$ Sample size. The sample size came to be 100 . We included 120 samples in our study. Informed consent was obtained from parents or patients before recording data as applicable. The patients were examined in a clinical set up of dental OPD with illuminated light using mouth mirror, probe and tweezers. The inclusion criteria were 1) Participants must be a resident of province II 2) 11-23 years of age 3) All set of permanent teeth erupted (except second and third molars). The exclusion criteria were subjects with 1) Proximal or occlusal wear, interproximal caries or restorations 2) Crowding, spacing, cross bite, over retained deciduous tooth, missing permanent tooth, any dental anomalies 3) Cleft lip and palate or any other syndromes 4) Past history of orthodontic treatment. After obtaining the consent from patients meeting the inclusion criteria, alginate impressions of both maxillary and mandibular teeth were made by a trained dental surgeon. The impressions were poured with dental stone, Type 3 gypsum product. Total numbers of participants were 120; 60 males and 60 females. Digital vernier calliper [0-150 mm, 799A-6/150, Starrett tools (Suzhou) Co. Ltd., China] providing measurements to $\pm 0.01 \mathrm{~mm}$ was used to measure the mesio-distal dimension of all teeth. The teeth measured included the mandibular and maxillary permanent incisors, permanent canines, first premolars, second premolars and first molars. All measurements were made by a single investigator. Maximum 10 pairs of casts measured per day to avoid eye fatigue. All measurements were done directly on study models. Maximum mesiodistal width of each tooth was measured and recorded to $0.1 \mathrm{~mm}$. All measurements were taken perpendicular to the long axis of the tooth with the calliper beak entering the inter-proximal area from the buccal or occlusal side. ${ }^{13}$ Repeated measurements were performed to minimize the possible errors. Intra-examiner reliability was predetermined at $0.2 \mathrm{~mm}$ as mentioned by Bishara et al. ${ }^{11}$ Bishara ${ }^{11,14}$ recommended that measurements that varied by $0.2 \mathrm{~mm}$ or less to be averaged and re-measurement was done for measurements that varied more than $0.2 \mathrm{~mm}$ and the three measurements were averaged.

Statistical analysis was performed with IBM SPSS Statistics Version 16 for Windows. Data were presented as mean, standard deviation (SD), frequency and percentage where appropriate. Independent t-test was used to compare between males and females. The significance level was set at $\mathrm{p}$ value $<=0.05$.

\section{RESULTS}

The data on mesio-distal crown dimensions of the permanent maxillary and mandibular teeth of the Nepalese population studied are summarized in table 1 and 2 respectively. The mean mesio-distal crown dimensions of the permanent dentition of males were larger than that of females for each type of tooth except maxillary central and lateral incisor. Comparison was done between the mean mesio-distal dimensions of right and left side of both maxillary and mandibular arches amongst females and males as shown in table 5 and 6 . The differences between the mean mesio-distal dimension of individual tooth on the right and left sides were comparable.

Table 1: Mesio-distal width of individual tooth in females

\begin{tabular}{lccc}
\multicolumn{1}{c}{ Tooth } & $\begin{array}{c}\text { Min width } \\
(\mathbf{m m})\end{array}$ & $\begin{array}{c}\text { Max width } \\
\mathbf{( m m )}\end{array}$ & $\begin{array}{c}\text { Mean+SD } \\
(\mathbf{m m})\end{array}$ \\
$\begin{array}{l}\text { Mandibular right first molar } \\
\begin{array}{l}\text { Mandibular right second } \\
\text { premolar }\end{array}\end{array}$ & 10.00 & 10.99 & $10.57 \pm 0.24$ \\
$\begin{array}{l}\text { Mandibular right first pre- } \\
\text { molar }\end{array}$ & 5.76 & 7.87 & $6.82 \pm 0.51$ \\
$\begin{array}{l}\text { Mandibular right canine } \\
\begin{array}{l}\text { Mandibular right lateral } \\
\text { incisor }\end{array}\end{array}$ & 5.41 & 7.71 & $6.91 \pm 0.48$ \\
$\begin{array}{l}\text { Mandibular right central } \\
\text { incisor }\end{array}$ & 4.63 & 7.93 & $6.65 \pm 0.45$ \\
Mandibular left central incisor & 4.12 & 6.82 & $5.87 \pm 0.42$ \\
\end{tabular}




\begin{tabular}{llll} 
Mandibular left lateral incisor & 4.82 & 6.90 & $5.87 \pm 0.41$ \\
Mandibular left canine & 5.50 & 7.96 & $6.62 \pm 0.47$ \\
Mandibular left first premolar & 5.31 & 7.70 & $6.89 \pm 0.48$ \\
$\begin{array}{l}\text { Mandibular left second } \\
\text { premolar }\end{array}$ & 5.73 & 8.03 & $6.86 \pm 0.52$ \\
Mandibular left first molar & 10.11 & 11.12 & $10.59 \pm 0.24$ \\
Maxillary right first molar & 9.15 & 9.97 & $9.59 \pm 0.22$ \\
Maxillary right second & 5.36 & 7.52 & $6.45 \pm 0.47$ \\
premolar & 5.22 & 7.77 & $6.84 \pm 0.48$ \\
Maxillary right first premolar & 6.48 & 8.43 & $7.57 \pm 0.42$ \\
Maxillary right canine & 6.08 & 7.23 & $6.62 \pm 0.27$ \\
Maxillary right lateral incisor & 8.09 & 8.97 & $8.51 \pm 0.23$ \\
Maxillary right central incisor & 8.13 & 9.09 & $8.53 \pm 0.25$ \\
Maxillary left central incisor & 6.12 & 7.30 & $6.64 \pm 0.27$ \\
Maxillary left lateral incisor & 6.65 & 8.40 & $7.59 \pm 0.40$ \\
Maxillary left canine & 5.21 & 7.77 & $6.83 \pm 0.48$ \\
Maxillary left first premolar & 5.45 & 7.52 & $6.44 \pm 0.45$ \\
Maxillary left second premolar & 9.17 & 10.23 & $9.64 \pm 0.26$ \\
\hline Maxillary left first molar & & &
\end{tabular}

Table 2: Mesio-distal width of individual tooth in males

\begin{tabular}{|c|c|c|c|}
\hline Tooth & $\begin{array}{l}\text { Min width } \\
\text { (mm) }\end{array}$ & $\begin{array}{c}\text { Max width } \\
\text { (mm) }\end{array}$ & $\begin{array}{c}\text { Mean+SD } \\
(\mathrm{mm})\end{array}$ \\
\hline Mandibular right first molar & 9.60 & 12.15 & $10.94 \pm 0.61$ \\
\hline $\begin{array}{l}\text { Mandibular right second } \\
\text { premolar }\end{array}$ & 5.79 & 8.15 & $6.92 \pm 0.48$ \\
\hline $\begin{array}{l}\text { Mandibular right first pre- } \\
\text { molar }\end{array}$ & 5.89 & 8.31 & $7.03 \pm 0.46$ \\
\hline Mandibular right canine & 6.20 & 7.76 & $6.89 \pm 0.35$ \\
\hline Mandibular right lateral incisor & 4.97 & 6.70 & $6.04 \pm 0.36$ \\
\hline $\begin{array}{l}\text { Mandibular right central } \\
\text { incisor }\end{array}$ & 4.67 & 6.26 & $5.36 \pm 0.33$ \\
\hline Mandibular left central incisor & 4.66 & 6.26 & $5.37 \pm 0.33$ \\
\hline Mandibular left lateral incisor & 4.97 & 6.80 & $6.03 \pm 0.36$ \\
\hline Mandibular left canine & 6.20 & 7.77 & $6.92 \pm 0.33$ \\
\hline Mandibular left first premolar & 5.92 & 8.30 & $7.05 \pm 0.46$ \\
\hline $\begin{array}{l}\text { Mandibular left second } \\
\text { premolar }\end{array}$ & 5.79 & 8.05 & $6.88 \pm 0.46$ \\
\hline Mandibular left first molar & 9.79 & 12.03 & $10.97 \pm 0.60$ \\
\hline Maxillary right first molar & 9.92 & 11.14 & $10.49 \pm 0.31$ \\
\hline $\begin{array}{l}\text { Maxillary right second pre- } \\
\text { molar }\end{array}$ & 5.49 & 7.32 & $6.49 \pm 0.39$ \\
\hline Maxillary right first premolar & 5.82 & 7.85 & $6.98 \pm 0.38$ \\
\hline Maxillary right canine & 7.15 & 8.82 & $7.88 \pm 0.37$ \\
\hline Maxillary right lateral incisor & 5.91 & 7.14 & $6.52 \pm 0.33$ \\
\hline Maxillary right central incisor & 7.80 & 9.13 & $8.38 \pm 0.38$ \\
\hline Maxillary left central incisor & 7.50 & 9.16 & $8.37 \pm 0.38$ \\
\hline Maxillary left lateral incisor & 5.88 & 7.10 & $6.53 \pm 0.32$ \\
\hline Maxillary left canine & 7.16 & 8.82 & $7.86 \pm 0.35$ \\
\hline Maxillary left first premolar & 6.07 & 7.85 & $6.97 \pm 0.38$ \\
\hline Maxillary left second premolar & 5.29 & 7.89 & $6.48 \pm 0.43$ \\
\hline Maxillary left first molar & 9.97 & 11.09 & $10.49 \pm 0.29$ \\
\hline
\end{tabular}

Table 3: Comparison of tooth width of mandibular arch between female and male

\begin{tabular}{|c|c|c|c|c|}
\hline Tooth & Gender & $\begin{array}{c}\text { Mean } \pm \text { SD } \\
(\mathrm{mm})\end{array}$ & $\begin{array}{l}\text { S.E. } \\
\text { Mean }\end{array}$ & $P$ Value \\
\hline \multirow{2}{*}{$\begin{array}{l}\text { Mandibular right first } \\
\text { molar }\end{array}$} & $\mathrm{F}$ & $10.57 \pm 0.24$ & 0.031 & \multirow{2}{*}{$\mathrm{P}<0.000^{*}$} \\
\hline & M & $10.94 \pm 0.61$ & 0.079 & \\
\hline \multirow{2}{*}{$\begin{array}{l}\text { Mandibular right sec- } \\
\text { ond premolar }\end{array}$} & $\mathrm{F}$ & $6.82 \pm 0.51$ & 0.066 & \multirow{2}{*}{$P=0.281$} \\
\hline & M & $6.92 \pm 0.48$ & 0.061 & \\
\hline \multirow{2}{*}{$\begin{array}{l}\text { Mandibular right first } \\
\text { premolar }\end{array}$} & $\mathrm{F}$ & $6.91 \pm 0.48$ & 0.061 & \multirow{2}{*}{$P=0.156$} \\
\hline & M & $7.03 \pm 0.46$ & 0.060 & \\
\hline \multirow{2}{*}{ Mandibular right canine } & $\mathrm{F}$ & $6.65 \pm 0.45$ & 0.058 & \multirow{2}{*}{$\mathrm{P}=0.002^{*}$} \\
\hline & M & $6.89 \pm 0.35$ & 0.044 & \\
\hline \multirow{2}{*}{$\begin{array}{l}\text { Mandibular right lateral } \\
\text { incisor }\end{array}$} & $\mathrm{F}$ & $5.87 \pm 0.42$ & 0.054 & \multirow{2}{*}{$\mathrm{P}=0.017^{*}$} \\
\hline & M & $6.04 \pm 0.35$ & 0.045 & \\
\hline \multirow{2}{*}{$\begin{array}{l}\text { Mandibular right cen- } \\
\text { tral incisor }\end{array}$} & $\mathrm{F}$ & $5.35 \pm 0.44$ & 0.056 & \multirow{2}{*}{$\mathrm{P}=0.894$} \\
\hline & M & $5.36 \pm 0.34$ & 0.043 & \\
\hline \multirow{2}{*}{$\begin{array}{l}\text { Mandibular left central } \\
\text { incisor }\end{array}$} & $\mathrm{F}$ & $5.33 \pm 0.42$ & 0.054 & \multirow{2}{*}{$P=0.556$} \\
\hline & M & $5.37 \pm 0.33$ & 0.042 & \\
\hline \multirow{2}{*}{$\begin{array}{l}\text { Mandibular left lateral } \\
\text { incisor }\end{array}$} & $\mathrm{F}$ & $5.87 \pm 0.41$ & 0.052 & \multirow{2}{*}{$\mathrm{P}=0.032^{*}$} \\
\hline & M & $6.03 \pm 0.36$ & 0.047 & \\
\hline \multirow{2}{*}{ Mandibular left canine } & $\mathrm{F}$ & $6.62 \pm 0.47$ & 0.060 & \multirow{2}{*}{$\mathrm{P}<0.000^{*}$} \\
\hline & M & $6.92 \pm 0.33$ & 0.042 & \\
\hline \multirow{2}{*}{$\begin{array}{l}\text { Mandibular left first } \\
\text { premolar }\end{array}$} & $\mathrm{F}$ & $6.89 \pm 0.48$ & 0.062 & \multirow{2}{*}{$\mathrm{P}=0.084$} \\
\hline & M & $7.05 \pm 0.46$ & 0.058 & \\
\hline \multirow{2}{*}{$\begin{array}{l}\text { Mandibular left second } \\
\text { premolar }\end{array}$} & $\mathrm{F}$ & $6.87 \pm 0.52$ & 0.066 & \multirow{2}{*}{$P=0.788$} \\
\hline & M & $6.88 \pm 0.46$ & 0.059 & \\
\hline \multirow{2}{*}{$\begin{array}{l}\text { Mandibular left first } \\
\text { molar }\end{array}$} & $\mathrm{F}$ & $10.59 \pm 0.24$ & 0.031 & \multirow{2}{*}{$\mathrm{P}<0.000^{*}$} \\
\hline & M & $10.97 \pm 0.60$ & 0.077 & \\
\hline \multicolumn{5}{|c|}{$\mathrm{F}=$ female, $\mathrm{M}=$ male, ${ }^{*} \mathrm{p}<0.05$ significant } \\
\hline
\end{tabular}

Table 4: Comparison of tooth width of maxillary arch between male and female

\begin{tabular}{|c|c|c|c|c|}
\hline Tooth & Gender & $\begin{array}{l}\operatorname{Mean} \pm \text { SD } \\
(\mathrm{mm})\end{array}$ & $\begin{array}{l}\text { S.E. } \\
\text { Mean }\end{array}$ & $P$ Value \\
\hline \multirow{2}{*}{$\begin{array}{l}\text { Maxillary right first } \\
\text { molar }\end{array}$} & $\mathrm{F}$ & $9.59 \pm 0.22$ & 0.028 & \multirow{2}{*}{$\mathrm{P}<0.001$} \\
\hline & M & $10.49 \pm 0.31$ & 0.039 & \\
\hline \multirow{2}{*}{$\begin{array}{l}\text { Maxillary right second } \\
\text { premolar }\end{array}$} & $\mathrm{F}$ & $6.45 \pm 0.47$ & 0.061 & \multirow{2}{*}{$P=0.660$} \\
\hline & M & $6.49 \pm 0.39$ & 0.051 & \\
\hline \multirow{2}{*}{$\begin{array}{l}\text { Maxillary right first } \\
\text { premolar }\end{array}$} & $\mathrm{F}$ & $6.84 \pm 0.48$ & 0.061 & \multirow{2}{*}{$P=0.087$} \\
\hline & M & $6.98 \pm 0.38$ & 0.048 & \\
\hline \multirow{2}{*}{ Maxillary right canine } & $\mathrm{F}$ & $7.57 \pm 0.42$ & 0.054 & \multirow{2}{*}{$\mathrm{P}<0.000^{*}$} \\
\hline & M & $7.88 \pm 0.37$ & 0.048 & \\
\hline \multirow{2}{*}{$\begin{array}{l}\text { Maxillary right lateral } \\
\text { incisor }\end{array}$} & $\mathrm{F}$ & $6.62 \pm 0.27$ & 0.035 & \multirow{2}{*}{$\mathrm{P}=0.071$} \\
\hline & M & $6.52 \pm 0.33$ & 0.042 & \\
\hline \multirow{2}{*}{$\begin{array}{l}\text { Maxillary right central } \\
\text { incisor }\end{array}$} & $\mathrm{F}$ & $8.51 \pm 0.23$ & 0.029 & \multirow{2}{*}{$\mathrm{P}=0.020^{*}$} \\
\hline & M & $8.38 \pm 0.38$ & 0.048 & \\
\hline \multirow{2}{*}{$\begin{array}{l}\text { Maxillary left central } \\
\text { incisor }\end{array}$} & $\mathrm{F}$ & $8.53 \pm 0.25$ & 0.032 & \multirow{2}{*}{$\mathrm{P}=0.005^{*}$} \\
\hline & M & $8.37 \pm 0.38$ & 0.049 & \\
\hline \multirow{2}{*}{$\begin{array}{l}\text { Maxillary left lateral } \\
\text { incisor }\end{array}$} & $\mathrm{F}$ & $6.64 \pm 0.27$ & 0.035 & \multirow{2}{*}{$\mathrm{P}=0.032^{*}$} \\
\hline & M & $6.53 \pm 0.32$ & 0.041 & \\
\hline \multirow{2}{*}{ Maxillary left canine } & $\mathrm{F}$ & $7.59 \pm 0.40$ & 0.052 & \multirow{2}{*}{$\mathrm{P}<0.001^{*}$} \\
\hline & M & $7.86 \pm 0.35$ & 0.045 & \\
\hline
\end{tabular}




\begin{tabular}{lcccc}
$\begin{array}{lccc}\text { Maxillary left first } \\
\text { premolar }\end{array}$ & F & $6.84 \pm 0.48$ & 0.062 & \\
& M & $6.97 \pm 0.38$ & 0.049 & $\mathrm{P}=0.098$ \\
$\begin{array}{l}\text { Maxillary left second } \\
\text { premolar }\end{array}$ & F & $6.44 \pm 0.45$ & 0.057 & \\
& M & $6.48 \pm 0.43$ & 0.055 & $\mathrm{P}=0.624$ \\
Maxillary left first molar & $\mathrm{F}$ & $9.64 \pm 0.26$ & 0.033 & \\
& $\mathrm{M}$ & $10.49 \pm 0.29$ & 0.038 & $\mathrm{P}<0.001^{*}$ \\
$\mathrm{~F}=$ female, $\mathrm{M}=$ male, ${ }^{*} \mathrm{p}<0.05$ significant & & & \\
\hline
\end{tabular}

Table 5: Comparison of tooth width between right and left side of both arches in females

\begin{tabular}{|c|c|c|c|c|}
\hline Arch & Tooth notation & $\begin{array}{l}\text { Right }(\mathrm{mm}) \\
\text { Mean+SD } \\
(\mathrm{n}=60)\end{array}$ & $\begin{array}{c}\text { Left }(\mathrm{mm}) \\
\text { Mean+SD } \\
(\mathrm{n}=60)\end{array}$ & p-value \\
\hline \multirow{9}{*}{ Maxillary } & First Molar & $9.64 \pm 0.26$ & $9.59 \pm 0.23$ & 0.315 \\
\hline & Second Premolar & $6.44 \pm 0.45$ & $6.45 \pm 0.47$ & 0.924 \\
\hline & First Premolar & $6.84+0.48$ & $6.84+0.48$ & 0.937 \\
\hline & Canine & $7.59 \pm 0.40$ & $7.57 \pm 0.42$ & 0.783 \\
\hline & Lateral Incisor & $6.64 \pm 0.27$ & $6.62 \pm 0.27$ & 0.670 \\
\hline & Central Incisor & $8.53 \pm 0.25$ & $8.51 \pm 0.23$ & 0.577 \\
\hline & First Molar & $10.59 \pm 0.24$ & $10.57 \pm 0.24$ & 0.634 \\
\hline & Second Premolar & $6.86 \pm 0.52$ & $6.82 \pm 0.51$ & 0.698 \\
\hline & First Premolar & $6.89 \pm 0.82$ & $6.91 \pm 0.48$ & 0.909 \\
\hline \multirow{3}{*}{ Mandibular } & Canine & $6.62 \pm 0.0 .47$ & $6.65 \pm 0.45$ & 0.720 \\
\hline & Lateral Incisor & $5.87 \pm 0.41$ & $5.87 \pm 0.42$ & 0.942 \\
\hline & Central Incisor & $5.33 \pm 0.43$ & $5.35 \pm 0.44$ & 0.769 \\
\hline & & & & \\
\hline
\end{tabular}

Table 6: Comparison of tooth width between right and left side of both arches in males

\begin{tabular}{|c|c|c|c|c|}
\hline Arch & Tooth notation & $\begin{array}{c}\text { Right(mm) } \\
\text { Mean+SD } \\
(\mathrm{n}=60)\end{array}$ & $\begin{array}{c}\text { Left }(\mathrm{mm}) \\
\text { Mean+SD } \\
(\mathrm{n}=60)\end{array}$ & p-value \\
\hline \multirow{9}{*}{ Maxillary } & First Molar & $10.49 \pm 0.29$ & $10.49 \pm 0.31$ & 0.990 \\
\hline & Second Premolar & $6.48 \pm 0.43$ & $6.49 \pm 0.39$ & 0.960 \\
\hline & First Premolar & $6.97 \pm 0.38$ & $6.98 \pm 0.38$ & 0.880 \\
\hline & Canine & $7.86 \pm 0.35$ & $7.88 \pm 0.37$ & 0.837 \\
\hline & Lateral Incisor & $6.53 \pm 0.32$ & $6.52 \pm 0.33$ & 0.953 \\
\hline & Central Incisor & $8.37 \pm 0.38$ & $8.38 \pm 0.38$ & 0.897 \\
\hline & First Molar & $10.97 \pm 0.60$ & $10.94 \pm 0.61$ & 0.813 \\
\hline & Second Premolar & $6.88 \pm 0.46$ & $6.92 \pm 0.48$ & 0.663 \\
\hline & First Premolar & $7.05 \pm 0.46$ & $7.03 \pm 0.46$ & 0.842 \\
\hline \multirow{3}{*}{ Mandibular } & Canine & $6.91 \pm 0.33$ & $6.89 \pm 0.35$ & 0.674 \\
\hline & Lateral Incisor & $6.03 \pm 0.36$ & $6.04 \pm 0.36$ & 0.843 \\
\hline & Central Incisor & $5.37 \pm 0.33$ & $5.36 \pm 0.34$ & 0.891 \\
\hline${ }^{*} \mathrm{p}<0.05 \mathrm{sign}$ & ficant & & & \\
\hline
\end{tabular}

The largest sexual dimorphism in mesio-distal crown dimension was exhibited by the maxillary molars $(0.88 \mathrm{~mm})$ followed by mandibular molars $(0.38 \mathrm{~mm})$, similarly in anterior tooth segment the maxillary canines $(0.29 \mathrm{~mm})$ followed by the mandibular canines $(0.27 \mathrm{~mm})$.

In both genders the maxillary first premolars exhibits greater width than the maxillary second premolars, averaging $0.42 \mathrm{~mm}$. In the mandibular arch, the lateral incisors were wider than the central incisors, by an average dimension of $0.67 \mathrm{~mm}$ in males and $0.53 \mathrm{~mm}$ in females. In the mandibular arch, the mean mesio-distal crown dimension of the central incisors was less than that of the lateral incisors for both the genders. In mandibular arch, the mean mesio-distal crown dimension of the first premolars was greater than that of the second premolars for males but almost same for females. In both males and females, the SD of tooth size measurement showed that variability differed between individual teeth.

\section{DISCUSSION}

In the present study, the mean mesio-distal width of the individual tooth showed larger tooth size in males as compared to females except for maxillary central and lateral incisor. This is similar to the study done in Nepalese population by Shrestha $\mathrm{R}^{15}$ in Bangladeshi population by Khan et al. ${ }^{16}$ The study showed that teeth in males, with the exception of the mandibular $2^{\text {nd }}$ premolar, mandibular $1^{\text {st }}$ premolar ,mandibular central incisors, maxillary $2^{\text {nd }}$ premolar, maxillary $1^{\text {st }}$ premolar ,maxillary right lateral incisors, had significantly greater mesio-distal crown width than those in females $\mathrm{p}$ value $<=0.05$ table 3 and 4 ,which is similar as reported by Kundi ${ }^{17}$ in his study.

There were no significant differences in the mesio-distal dimensions as compared to the right and left side in both females and males as shown in table 5 and 6 . This was similar to the studies done by Khan et $\mathrm{al}^{16}$ in Bangladeshi population. This result is in contrary to the study done by Shrestha $\mathrm{R}^{15}$ in Nepalese population, which showed significant differences in the size of maxillary first molars, maxillary second molars, mandibular central incisors and mandibular lateral incisors, which were larger in right side. These differences could be due to the variation in distribution of different ethnic groups in samples collected. Mahmoud NM ${ }^{4}$, Lavelle et al. ${ }^{1}$ and Lundstoum ${ }^{18}$ also found definite differences between right and left side teeth measurements.

The results obtained in this study will provide useful clinical information to diagnose and frame treatment planning for orthodontic patients of province II of Nepal. This will help orthodontist to achieve good treatment results. As Nepal is a country with ethnic diversity this study can be extended to other provinces with larger sample size.

\section{CONCLUSIONS}

From the findings, it could be concluded that, the differences between the mean mesio-distal dimension 
of individual tooth on the right and left sides were comparable. The mean mesio-distal crown dimensions of the permanent dentition of males were larger than that of females for each type of tooth except maxillary central and lateral incisor.

\section{CONFLICT OF INTEREST}

None declared

\section{FUNDING}

No any funding source.

\section{REFERENCES}

1. Lavelle CL. Maxillary and mandibular tooth size in different racial groups and in different occlusal categories. Am J Orthod. 1972 Jan; 61(1):29-37. DOI: 10.1016/0002-9416(72)90173-X

2. Proffit W. Contemporary orthodontics. 4th ed. St. Louis: Mosby; 2007. pp. 195-201.

3. Mulimani PS, Azmi MI, Jamali NR, Basir NN, Soe HH. Bolton's tooth size discrepancy in Malaysian orthodontic patients: Are occlusal characteristics such as overjet, overbite, midline, and crowding related to tooth size discrepancy in specific malocclusions and ethnicities?. APOS Trends Orthod 2018;8:36-43. DOI: 10.4103/apos.apos_104_17

4. Mahmoud NM, Eltahir HE, Mageet AO. Tooth Size Discrepancy among Different Malocclusion Groups in a Sudanese Sample. J Orthod Endod 2017;3 (2):10. DOI: $10.21767 / 2469-2980.100044$

5. Crosby DR, Alexander CG. The occurrence of tooth size discrepancies among different malocclusion groups. Am J Orthod Dentofacial Orthop. 1989 Jun; 95 (6):457-61. DOI: 10.1016/0889-5406(89)90408-3

6. Freeman JE, Maskeroni AJ, Lorton L. Frequency of Bolton tooth-size discrepancies among orthodontic patients. Am J Orthod Dentofacial Orthop. 1996 Jul; 110(1):24-7. DOI: 10.1016/S0889-5406(96)70083-5

7. Arya BS, Savara BS, Thomas D, Clarkson Q. Relation of sex and occlusion to mesiodistal tooth size. Am J Orthod. 1974 Nov; 66(5):479-86. DOI: 10.1016/00029416(74)90109-2

8. Ta TA, Ling JY, Hägg U. Tooth-size discrepancies among different occlusion groups of southern Chinese children. Am J Orthod Dentofacial Orthop. 2001 Nov; 120(5):556-8. DOI: $\quad 10.1067 / \bmod .2001 .118998$ PMID: 11709674.

9. Buschang PH, Demirjian A, Cadotte L. Permanent mesiodistal tooth size of French-Canadians. J Can Dent
Assoc. 1988 Jun; 54(6):441-4.

10. Smith SS, Buschang PH, Watanabe E. Interarch tooth size relationships of 3 populations: "does Bolton's analysis apply?" Am J Orthod Dentofacial Orthop. 2000 Feb; 117(2):169-74. DOI: 10.1016/S08895406(00)70228-9

11. Bishara SE, Jakobsen JR, Abdallah EM, Fernandez Garcia A. Comparisons of mesiodistal and buccolingual crown dimensions of the permanent teeth in three populations from Egypt, Mexico, and the United States. Am J Orthod Dentofacial Orthop. 1989 Nov; 96(5):416-22. DOI: 10.1016/0889-5406(89)90326-0

12. Shirazi S, Kachoei M, Shahvaghar-Asl N, Shirazi S, Sharghi R. Arch width changes in patients with Class II division 1 malocclusion treated with maxillary first premolar extraction and non-extraction method. J Clin Exp Dent. 2016;8(4):e403-8. DOI: 10.4317/ jced.52840 PMID: 27703608.

13. Legović M, Novosel A, Legović A. Regression equations for determining mesiodistal crown diameters of canines and premolars. Angle Orthod. 2003 Jun; 73(3):314-8.

14. Bishara SE, Fernandez Garcia A, Jakobsen JR, Fahl JA. Mesiodistal crown dimensions in Mexico and the United States. Angle Orthod. 1986 Oct; 56(4):315-23.

15. Shrestha R. Measurement of mesio-distal tooth diameter of Nepalese permanent dentition. J Nep Dent Assoc. 2005; 7(1):55-63.

16. Khan SA, Hassan GS, Rafique T, Hasan MN. Md. Hossain Russell M S. Mesiodistal Crown Dimensions of Permanent Teeth in Bangladeshi Population. BSMMU J. 2011; 4(2):81-7. DOI: 10.3329/bsmmuj.v4i2.8635

17. Kundi I. Mesiodistal crown dimensions of the permanent dentitions in different malocclusions in Saudi populations: an aid in sex determinations. Pak Oral Dent J. 2015 Sep; 35(3):429-33.

18. Lundstrom A. Intermaxillary tooth width ratio and tooth alignment and occlusion. Acta Odontol Scand. 1955 Feb; 12(3-4):265-92. DOI: 10.3109/00016355509028167 PMID:14375894. 